\title{
Implementation of a separate fluid neutral energy equation in SOLPS-ITER and its impact on the validity range of advanced fluid neutral models
}

\author{
W. Van Uytven ${ }^{1}$, M. Blommaert ${ }^{1}$, W. Dekeyser ${ }^{1}$, N. Horsten ${ }^{1}$, and M. Baelmans ${ }^{1}$ \\ ${ }^{1}$ Department of Mechanical Engineering, KU Leuven
}

\begin{abstract}
In plasma edge transport codes for nuclear fusion devices, fluid neutral models offer an interesting alternative to the currently used kinetic Monte Carlo simulations, especially for cases of high ion-neutral collisionality. In this paper, we elaborate a separate neutral energy equation in the state-of-the-art SOLPS-ITER code suite, which previously assumed perfect ion-neutral temperature equilibration. Furthermore, we study the coupled plasma-neutral solutions for a range of divertor operating regimes, proving the validity of these fluid neutral models for high-recycling and detached regimes.
\end{abstract}

\section{Introduction}

Plasma edge transport codes such as SOLPS-ITER [13 are intensively used for the analysis of plasma edge physics in existing tokamaks and for the design of future fusion devices. These codes typically use a finitevolume (FV) discretization for the plasma, which is modeled using fluid equations with parallel transport based on Braginskii [3] and diffusive anomalous transport perpendicular to the magnetic field lines, coupled to a kinetic Monte Carlo (MC) code for the neutral particles. In the case of SOLPS-ITER, EIRENE [11] is used as MC code. According to current knowledge, future fusion reactors such as ITER will require a (partially) detached divertor operation to decrease the peak target heat loads to acceptable levels [10]. In these detached regimes, the neutral hydrogenic species undergo a large amount of charge-exchange $(\mathrm{CX})$ collisions with the ions. This high collisionality makes the MC code computationally expensive. Furthermore, the statistical noise from the MC part severely complicates convergence of the coupled plasma-neutral simulation. On the other hand, a high number of collisions implies that a fluid approach for the neutrals becomes valid. Fluid approximations for the neutral particles are already in use for decades 14, 12, showing qualitative agreement with the kinetic description in regimes of high ion-neutral collisionality. Recent developments of N. Horsten et al. 8 to make the boundary conditions and transport coefficients more consistent with the kinetic counterpart have led to a stronger quantitative agreement between the fluid and kinetic solutions, at least for the hydrogenic atoms. The accuracy of these models in the SOLPS-ITER code suite was studied by M. Blommaert et al. [2], confirming their high accuracy in detached regimes. The implementation, however, assumed perfect equilibration between ion and neutral temperatures. Although we expect this equilibration to be almost perfect in regions with a very high charge-exchange collisionality, a finite ion-neutral temperature difference can be observed in large areas of the divertor in the kinetic EIRENE simulations, even in case of high-recycling and detached regimes. In a first comparison by Horsten et al. [8] of the neutral models on a fixed background plasma, a separate neutral energy equation has shown to significantly reduce the fluid-kinetic discrepancy for the ion energy source terms. 
Until now, the assessment of the advanced fluid neutral models, developed by N. Horsten et al., was mostly limited to a comparison of the MC plasma sources with those of the fluid neutral model on a fixed plasma background. Although this approach is fast and valuable, it remains unknown to what extent differences in plasma sources will affect the fully converged coupled plasma-neutral solution. Thus far, only for a fully detached regime excellent agreement for the coupled solution has been shown 1].

In this contribution, we develop a separate fluid neutral energy equation in the state-of-the-art SOLPSITER plasma edge code suite and we benchmark this novel fluid model with the EIRENE MC code. Furthermore, we compare the coupled plasma-neutral solutions for a range of cases spanning from medium-recycling to fully detached, either using fluid neutral models (with and without the novel separate neutral energy equation) or the traditional MC simulation of the neutrals.

Section 2 presents an overview of the fluid neutral model equations, with focus on the existing combined ion-neutral energy equation and the novel separate ion and neutral energy equations. Section 3 details the geometry and plasma model used in this study. In Section 4, the plasma sources from both fluid models are compared with those from the EIRENE kinetic neutral simulation and the agreement for the coupled solutions is assessed for a range of operating regimes.

\section{Fluid neutral model equations}

This section presents an overview of the advanced fluid neutral model equations in SOLPS-ITER. We model a pure deuterium plasma and we neglect molecules in the plasma volume by assuming each molecule immediately dissociates at the walls with a Franck-Condon energy of $2 \mathrm{eV}[6]$. Before elaborating the separate neutral energy equation, we present the neutral continuity, parallel momentum and combined ion-neutral internal energy equation, which are unchanged with respect to the model of M. Blommaert et al.[2].

The neutral continuity equation is given by

$$
\frac{\partial n_{\mathrm{n}}}{\partial t}+\nabla \cdot \Gamma_{\mathrm{n}}^{n}=S_{\mathrm{n}}^{n}
$$

with the poloidal component $\Gamma_{\mathrm{n}, \theta}^{n}=n_{\mathrm{n}} b_{\theta} V_{\mathrm{n}, \|}-D_{\mathrm{n}}^{p} \nabla_{\theta} p_{\mathrm{n}}$ and the radial component $\Gamma_{\mathrm{n}, r}^{n}=-D_{\mathrm{n}}^{p} \nabla_{r} p_{\mathrm{n}}$. Here, $n_{\mathrm{n}}$ is the neutral density, $V_{\mathrm{n},||}$ is the neutral parallel velocity, $b_{\theta}$ is the magnetic field pitch and $p_{\mathrm{n}}=n_{\mathrm{n}} T_{\mathrm{n}}$ is the neutral pressure, with $T_{\mathrm{n}}$ the neutral temperature. $D_{\mathrm{n}}^{p}=\left(m\left(n_{\mathrm{i}} K_{\mathrm{cx}, m}+n_{\mathrm{e}} K_{\mathrm{i}}\right)\right)^{-1}$ is the pressure-diffusion coefficient, with $m$ the mass of the deuterium atom, $n_{\mathrm{i}}$ and $n_{\mathrm{e}}$ respectively the ion and electron density, $K_{\mathrm{i}}$ the ionization rate coefficient and $K_{\mathrm{cx}, m}$ the momentum-linearized CX rate coefficient, as defined in Ref. 9]. The neutral particle source term $S_{\mathrm{n}}^{n}$ is determined by the ionization and recombination processes, giving:

$$
S_{\mathrm{n}}^{n}=n_{\mathrm{i}} n_{\mathrm{e}} K_{\mathrm{r}}-n_{\mathrm{n}} n_{\mathrm{e}} K_{\mathrm{i}} .
$$

The neutral parallel momentum equation is given by

$$
m \frac{\partial n_{\mathrm{n}} V_{\mathrm{n}, \|}}{\partial t}+\nabla \cdot \Gamma_{\mathrm{n}}^{\mathrm{m}}+\nabla_{\|} p_{\mathrm{n}}=S_{\mathrm{n}, \|}^{\mathrm{m}}+S_{\mathrm{c}},
$$

with the neutral parallel momentum source given by

$$
S_{\mathrm{n}, \| \mid}^{\mathrm{m}}=m\left(\left(n_{\mathrm{i}} n_{\mathrm{e}} K_{\mathrm{r}}+n_{\mathrm{n}} n_{\mathrm{i}} K_{\mathrm{cx}, m}\right) V_{\mathrm{i}, \|}-\left(n_{\mathrm{n}} n_{\mathrm{e}} K_{\mathrm{i}}+n_{\mathrm{n}} n_{\mathrm{i}} K_{\mathrm{cx}, m}\right) V_{\mathrm{n}, \|}\right) .
$$

$S_{\mathrm{c}}$ represents curvature terms for which more details can be found in Ref. 8. The momentum fluxes are given by $\Gamma_{\mathrm{n}, \theta}^{\mathrm{m}}=m V_{\mathrm{n}, \|} \Gamma_{\mathrm{n}, \theta}^{n}-\eta_{\mathrm{n}, \theta \theta} \nabla_{\theta} V_{\mathrm{n}, \| \mid}$ and $\Gamma_{\mathrm{n}, r}^{\mathrm{m}}=m V_{\mathrm{n}, \|} \Gamma_{\mathrm{n}, r}^{n}-\eta_{\mathrm{n}, r r} \nabla_{r} V_{\mathrm{n}, \|}$. The isotropic neutral viscosity is given by $\eta_{\mathrm{n}, \theta \theta}=\eta_{\mathrm{n}, r r}=p_{\mathrm{n}} /\left(n_{\mathrm{i}} K_{\mathrm{cx}, m}\right)$.

The SOLPS-ITER code suite normally solves two internal energy equations: one for the electrons and one for all ion and fluid neutral species combined. For single-species cases without plasma drifts and currents, the combined ion-neutral internal energy equation reduces to:

$$
\sum_{a=\mathrm{i}, \mathrm{n}}\left[\frac{3}{2} \frac{\partial n_{a} T_{\mathrm{i}+\mathrm{n}}}{\partial t}+\nabla \cdot \Gamma_{a}^{\mathrm{H}}+p_{a} \nabla \cdot \mathbf{V}_{a, \|}-\nabla V_{a, \|} \cdot \eta_{a} \cdot \nabla V_{a, \|}\right]=Q_{\mathrm{ei}}+S_{\mathrm{i}+\mathrm{n}}^{\mathrm{H}},
$$


with

$$
\begin{gathered}
\Gamma_{a, \theta}^{\mathrm{H}}=-\kappa_{a, \theta \theta} \nabla_{\theta} T_{a}+\frac{5}{2} T_{a} \Gamma_{a, \theta}^{n}-T_{a} n_{a} V_{a, \|} b_{\theta}, \\
\Gamma_{a, r}^{\mathrm{H}}=-\kappa_{a, r r} \nabla_{r} T_{a}+\frac{5}{2} T_{a} \Gamma_{a, r}^{n}
\end{gathered}
$$

and

$$
S_{\mathrm{i}+\mathrm{n}}^{\mathrm{H}}=\frac{m}{2}\left(n_{\mathrm{n}} n_{\mathrm{e}} K_{\mathrm{i}}+n_{\mathrm{i}} n_{\mathrm{e}} K_{\mathrm{r}}+2 n_{\mathrm{n}} n_{\mathrm{i}} K_{\mathrm{cx}, m}\right)\left(V_{\mathrm{i}, \|}-V_{\mathrm{n}, \|}\right)^{2} .
$$

For the neutrals we have an isotropic heat conductivity $\kappa_{\mathrm{n}, \theta \theta}=\kappa_{\mathrm{n}, r r}=5 p_{\mathrm{n}} /\left(2 m\left(n_{\mathrm{i}} K_{\mathrm{cx}, m}\right)\right) . \quad \eta_{a}$ is the viscosity tensor, $Q_{\mathrm{ei}}$ is the ion-electron energy equilibration term and $S_{\mathrm{i}+\mathrm{n}}^{\mathrm{H}}$ is the combined ion-neutral heat source. This equation is solved for the combined ion-neutral temperature $T_{\mathrm{i}+\mathrm{n}}$, i.e. $T_{a}=T_{\mathrm{i}}=T_{\mathrm{n}}=T_{\mathrm{i}+\mathrm{n}}$.

In this contribution, we investigate whether the fluid-kinetic discrepancy can be further reduced by lifting the assumption of equal ion and neutral temperature in the SOLPS-ITER code suite. Separating the ion and neutral contributions from Eq. (5) leads to

$$
\frac{3}{2} \frac{\partial n_{\mathrm{i}} T_{\mathrm{i}}}{\partial t}+\nabla \cdot \boldsymbol{\Gamma}_{\mathrm{i}}^{\mathrm{H}}+p_{\mathrm{i}} \nabla \cdot \mathbf{V}_{\mathrm{i}, \|}-\nabla V_{\mathrm{i}, \|} \cdot \eta_{\mathrm{i}} \cdot \nabla V_{\mathrm{i}, \|}=Q_{\mathrm{ei}}+S_{\mathrm{i}}^{\mathrm{H}}
$$

and

$$
\frac{3}{2} \frac{\partial n_{\mathrm{n}} T_{\mathrm{n}}}{\partial t}+\nabla \cdot \boldsymbol{\Gamma}_{\mathrm{n}}^{\mathrm{H}}+p_{\mathrm{n}} \nabla \cdot \mathbf{V}_{\mathrm{n}, \|}-\nabla V_{\mathrm{n}, \|} \cdot \eta_{\mathrm{n}} \cdot \nabla V_{\mathrm{n}, \|}=S_{\mathrm{n}}^{\mathrm{H}} .
$$

$\Gamma_{\mathrm{i}}^{\mathrm{H}}$ and $\boldsymbol{\Gamma}_{\mathrm{n}}^{\mathrm{H}}$ are now based on $T_{\mathrm{i}}$ and $T_{\mathrm{n}}$, respectively, and Eq. 99 and Eq. 10 are solved for $T_{\mathrm{i}}$ and $T_{\mathrm{n}}$ separately.

To obtain the expressions for the separate ion and neutral internal heat sources, $S_{\mathrm{i}}^{\mathrm{H}}$ and $S_{\mathrm{n}}^{\mathrm{H}}$, we start from the total energy sources, which express transfer of thermal and kinetic energy between ions and neutrals due to ionization, recombination and charge exchange:

$$
S_{\mathrm{n}}^{\mathrm{E}}=-S_{\mathrm{i}}^{\mathrm{E}}=\left(n_{\mathrm{i}} n_{\mathrm{e}} K_{\mathrm{r}}+n_{\mathrm{n}} n_{\mathrm{i}} K_{\mathrm{cx}, m}\right)\left(\frac{3}{2} T_{\mathrm{i}}+\frac{m}{2} V_{\mathrm{i}, \|}^{2}\right)-\left(n_{\mathrm{n}} n_{\mathrm{e}} K_{\mathrm{i}}+n_{\mathrm{n}} n_{\mathrm{i}} K_{\mathrm{cx}, m}\right)\left(\frac{3}{2} T_{\mathrm{n}}+\frac{m}{2} V_{\mathrm{n}, \|}^{2}\right) .
$$

Because SOLPS-ITER solves an internal heat equation, the total energy sources $\left(S^{\mathrm{E}}\right)$ must be converted to internal heat sources $\left(S^{\mathrm{H}}\right)$. It can be shown (see e.g. Ref. [4]) that for each species $a$ we have

$$
S_{a}^{\mathrm{H}}=S_{a}^{\mathrm{E}}-V_{a, \|} S_{a, \|}^{\mathrm{m}}+\frac{m}{2} V_{a, \|}^{2} S_{a}^{n} .
$$

Filling in Eq. 22) and Eq. (4) and rearranging leads to the internal heat sources

$$
S_{\mathrm{i}}^{\mathrm{H}}=-n_{\mathrm{i}} n_{\mathrm{e}} K_{\mathrm{r}} \frac{3}{2} T_{\mathrm{i}}+n_{\mathrm{n}} n_{\mathrm{e}} K_{\mathrm{i}}\left(\frac{3}{2} T_{\mathrm{n}}+\frac{m}{2}\left(V_{\mathrm{i}, \|}-V_{\mathrm{n}, \|}\right)^{2}\right)+n_{\mathrm{n}} n_{\mathrm{i}} K_{\mathrm{cx}, m}\left(-\frac{3}{2} T_{\mathrm{i}}+\frac{3}{2} T_{\mathrm{n}}+\frac{m}{2}\left(V_{\mathrm{i}, \|}-V_{\mathrm{n}, \|}\right)^{2}\right)
$$

and

$$
S_{\mathrm{n}}^{\mathrm{H}}=n_{\mathrm{i}} n_{\mathrm{e}} K_{\mathrm{r}}\left(\frac{3}{2} T_{\mathrm{i}}+\frac{m}{2}\left(V_{\mathrm{i}, \|}-V_{\mathrm{n}, \|}\right)^{2}\right)-n_{\mathrm{n}} n_{\mathrm{e}} K_{\mathrm{i}} \frac{3}{2} T_{\mathrm{n}}+n_{\mathrm{n}} n_{\mathrm{i}} K_{\mathrm{cx}, m}\left(\frac{3}{2} T_{\mathrm{i}}-\frac{3}{2} T_{\mathrm{n}}+\frac{m}{2}\left(V_{\mathrm{i}, \|}-V_{\mathrm{n}, \|}\right)^{2}\right) .
$$

Note that summing $S_{\mathrm{n}}^{\mathrm{H}}$ and $S_{\mathrm{i}}^{\mathrm{H}}$ and assuming $T_{\mathrm{i}}=T_{\mathrm{n}}$ indeed leads to the currently implemented combined ion-neutral heat source, Eq (8).

The advanced fluid neutral boundary conditions derived in Ref. [8] are formulated for a total energy equation. Hence a conversion is needed to implement these boundary conditions consistently in SOLPSITER. This conversion follows from the definitions of the energy and heat fluxes [4], resulting in

$$
\Gamma_{\mathrm{BC}, \mathrm{n}, \theta}^{\mathrm{H}}=\Gamma_{\mathrm{BC}, \mathrm{n}, \theta}^{\mathrm{E}}-T_{\mathrm{n}} n_{\mathrm{n}} V_{\mathrm{n}, \|} b_{\theta}-\frac{m}{2} V_{\mathrm{n}, \|}^{2} \Gamma_{\mathrm{BC}, \mathrm{n}, \theta}^{n}+\frac{\eta_{\mathrm{n}, \theta \theta}}{2} \nabla_{\theta} V_{\mathrm{n}, \|}^{2}
$$


and

$$
\Gamma_{\mathrm{BC}, \mathrm{n}, r}^{\mathrm{H}}=\Gamma_{\mathrm{BC}, \mathrm{n}, r}^{\mathrm{E}}-\frac{m}{2} V_{\mathrm{n}, \|}^{2} \Gamma_{\mathrm{BC}, \mathrm{n}, r}^{n}+\frac{\eta_{\mathrm{n}, r r}}{2} \nabla_{r} V_{\mathrm{n}, \|}^{2} .
$$

The reader is referred to Refs. [8, ,2], and references therein, for the development of these advanced fluid neutral transport coefficients and boundary conditions.

When EIRENE is used, the fluid neutral density $n_{\mathrm{n}}$ is scaled with a very small number, effectively reducing the combined ion-neutral energy equation (Eq. 5 ) to the pure ion energy equation (Eq. 9p, implying that the resulting temperature is now the ion temperature $T_{\mathrm{i}}$. The ion particle $\left(S_{\mathrm{i}}^{n}=-S_{\mathrm{n}}^{n}\right)$, ion parallel momentum $\left(S_{\mathrm{i}, \| \mid}^{\mathrm{m}}=-S_{\mathrm{n},||}^{\mathrm{m}}\right)$ and ion heat $\left(S_{\mathrm{i}}^{\mathrm{H}}\right)$ sources are computed by the MC simulation instead of through Eqs. 22, (4) and $(13)$. The calculation of the electron energy source due to neutral interaction $\left(S_{\mathrm{e}}^{\mathrm{E}}\right)$ is identical to Ref. [2].

\section{Case set-up}

For sake of computational time, we limit this study to steady-state cases. The different neutral models that will be compared in this study are coupled to the same plasma model. Parallel plasma transport is based on Braginskii [3], but flux limited. We use an anomalous diffusion coefficient $D^{n}=1.0 \mathrm{~m}^{2} \mathrm{~s}^{-1}$, ion/electron heat conductivity coefficients $\chi_{\mathrm{i}}=\chi_{\mathrm{e}}=1.0 \mathrm{~m}^{2} \mathrm{~s}^{-1}$ and ion viscosity coefficient $\eta_{\mathrm{i}}=0.2 \mathrm{~m}^{2} \mathrm{~s}^{-1}$ to approximate turbulent transport. Drifts and currents are not modeled and the potential equation is not solved. At the core boundary a fixed heat flux of 4.18 MW is imposed, equally distributed among ions and electrons. The core ion density is varied between $1.5 \cdot 10^{19} \mathrm{~m}^{-3}$ and $6 \cdot 10^{19} \mathrm{~m}^{-3}$, as explained in the next section. At the targets, sheath boundary conditions are imposed for the ions and electrons and at the wall and private flux regions decay lengths are imposed for $T_{\mathrm{i}}, T_{\mathrm{e}}$ and $n_{\mathrm{i}}$. At the private flux regions $2 \%$ of the incident neutrals and ions are pumped away from the domain.

An orthogonal slab case is used, with a poloidal dimension representative of the Joint European Torus. The magnetic field pitch is spatially constant with $b_{\theta}=0.075$. The grid consists of 140 cells in the poloidal direction and 40 cells in the radial direction. The grid is poloidally refined towards the targets, where the plasma-neutral interaction is most important. The first interior cell has a poloidal width of $1.9 \mathrm{~mm}$. Figure 1 depicts the grid structure.

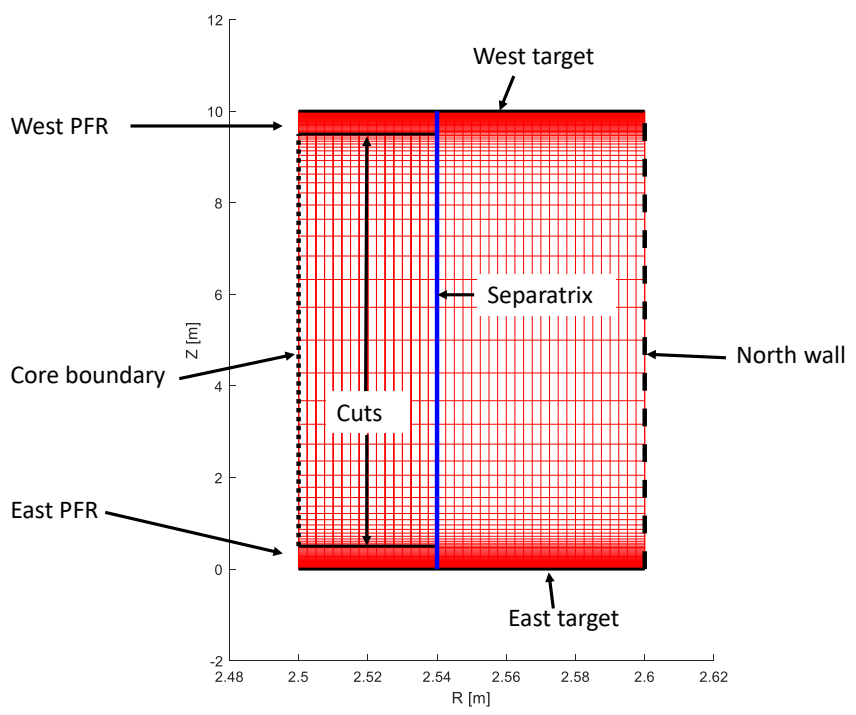

Figure 1: Computational mesh used in this study. The cuts separate the private flux region (PFR) from the core and connect both parts in physical space. Figure adapted from Ref. [2]. 


\section{Results}

We first compare the plasma sources due to ion-neutral interaction on a fixed plasma background. The plasma background is obtained by converging the coupled plasma-fluid neutral system using the combined ion-neutral energy equation. For the source term comparison, we select a high-recycling case with a core ion density of $2 \mathrm{e} 19 \mathrm{~m}^{-3}$. Figure 2 compares the ion particle source $S_{\mathrm{i}}^{n}$, the ion parallel momentum source $S_{\mathrm{i}, \|}^{\mathrm{m}}$, the electron energy source $S_{\mathrm{e}}^{\mathrm{E}}$ and the ion heat source $S_{\mathrm{i}}^{\mathrm{H}}$ as computed by the different neutral models. For the fluid neutral model with combined ion-neutral energy equation, the ion heat source $S_{\mathrm{i}}^{\mathrm{H}}$ is computed in a post-processing step using Eq. (11), but with $T_{\mathrm{i}}=T_{\mathrm{n}}=T_{\mathrm{i}+\mathrm{n}}$. Only the first $40 \mathrm{~mm}$ are shown, as this is where plasma-neutral interaction is most important.
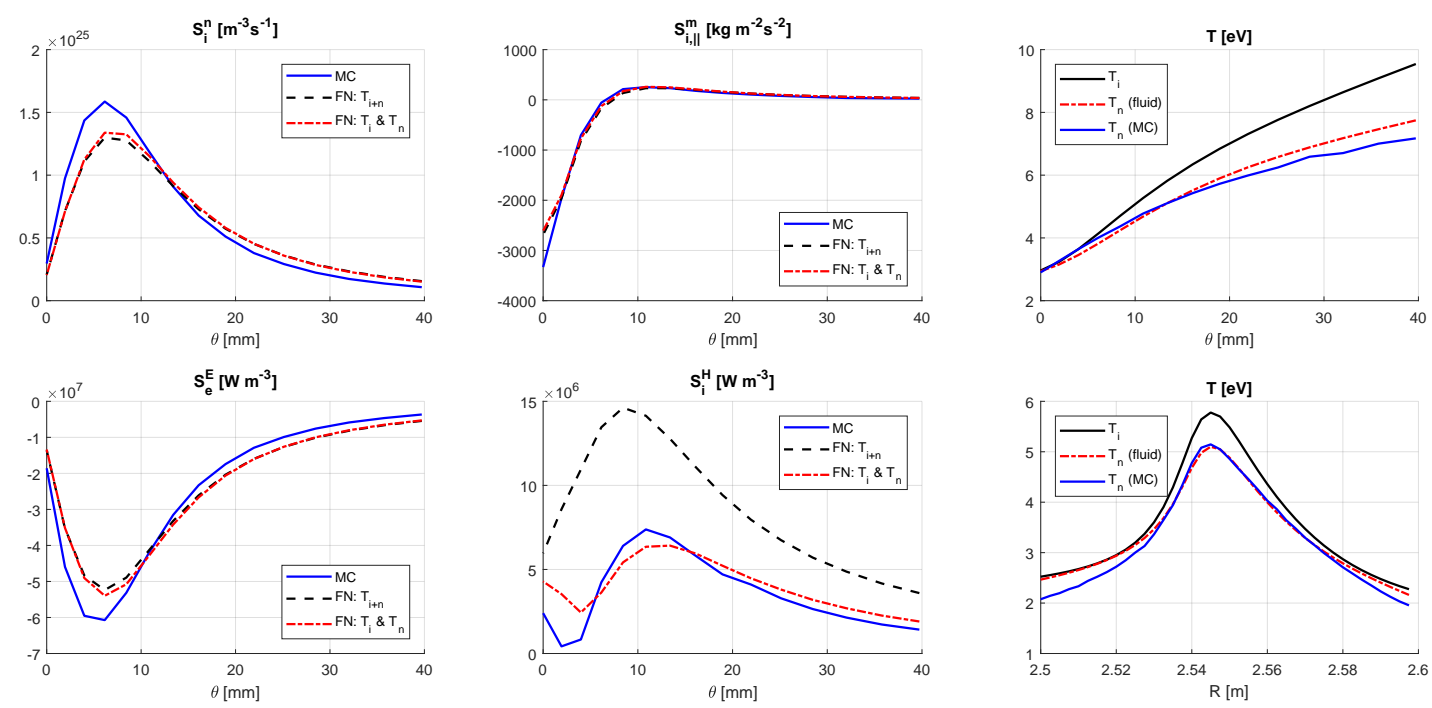

Figure 2: Comparison of the ion particle (top left), ion parallel momentum (top middle), electron energy (bottom left) and ion heat (bottom middle) sources. We compare the sources as computed by the EIRENE Monte Carlo code (blue line), the advanced fluid neutral (FN) model with combined ion-neutral energy equation (black dashed line) and the novel fluid model with separate neutral temperature (red dasheddotted line). The horizontal axis denotes the poloidal distance to the target. Results are displayed along the separatrix. The top right panel displays the ion temperature (black line), the neutral temperature as computed by EIRENE (blue line) and the neutral temperature as calculated by the fluid model (red dasheddotted line), along the separatrix. The bottom right panel provides radial temperature profiles, $10 \mathrm{~mm}$ in front of the target.

For this high-recycling case, the continuity and parallel momentum sources from both fluid models show good agreement with the EIRENE sources. The neutral continuity and momentum equation are influenced by the separate fluid neutral temperature through the neutral pressure, the viscosity $\eta_{n}$ and the linearized CX rate coefficient $K_{\mathrm{cx}, m}$. These dependencies are clearly weak for the case at hand as both fluid models give very similar results. The peak particle and electron heat source are slightly better matched with the separate neutral energy equation, but the improvement is limited compared to the remaining discrepancy with EIRENE. The discrepancies are $20 \%$ and $23 \%$ for the peak values of plasma particle and parallel momentum source, respectively. The ion heat source term, however, is substantially improved by solving a separate neutral energy equation. Upstream, the profiles match excellently, whereas assuming $T_{\mathrm{i}}=T_{\mathrm{n}}$ overestimates the ion heat source by more than $100 \%$. At the target there remains a substantial error for the novel fluid model, but there is still a significant qualitative and quantitative improvement over the use of the combined ion-neutral energy equation. This improvement is achieved because the ion-neutral temperature difference 
as calculated EIRENE can partially be captured by the separate fluid neutral energy equation. The top and bottom right panels of Figure 2 show a poloidal and radial profile of the ion and neutral temperatures. Close to the target, before the ionization peak $(\theta ; 5 \mathrm{~mm})$, ion-neutral temperature equilibration is very strong and the separate fluid neutral energy equation provides no improvement. Also, radially outward, the neutrals behave kinetically and their temperature cannot be captured with the fluid neutral model. However, in the viscinity of the separatrix $(R=2.54 \mathrm{~m})$ and upstream of the start of the ionization peak $(\theta ; 5 \mathrm{~mm})$, the fluid neutral energy equation captures the ion-neutral temperature differences excellently.

Next, we study the converged coupled plasma-neutral solutions. The pure fluid models are converged to machine precision. The fluid-kinetic system cannot be converged to machine precision due to the statistical noise from the MC simulations. As a compromise between speed and accuracy we use the random noise averaging procedure developed by K. Ghoos et al. [7]. We compare the different models over a range of operating regimes by varying only the core ion density and keeping all other model parameters fixed. Figure 3 shows the maximum target electron temperature and ion saturation current, for the different models and recycling levels.

The roll-over in ion saturation current is clearly visible in the top right panel of Figure 3 and qualitatively similar for all three neutral models. After the roll-over, the maximum target electron temperature remains roughly constant at $0.75 \mathrm{eV}$. When lowering the core density, the temperatures at the target start to increase significantly. For high-recycling and detached cases, the fluid models provide an adequate alternative to the standard MC approach. Modeling a separate neutral temperature improves results even further. For the case with lowest core ion density, $n_{\mathrm{i}, \mathrm{c}}=1.5 \cdot 10^{19} \mathrm{~m}^{-3}$, the fluid neutral model is clearly not valid anymore. While the error on the ion saturation current remains within $40 \%$, the error on the target electron temperatures becomes unacceptable. With a separate neutral energy equation, the maximum relative error is $12.8 \%$ for the maximum electron temperature and $7.3 \%$ for the ion saturation current, for high-recycling and detached regimes $\left(n_{\mathrm{i}, \mathrm{c}}=2 \cdot 10^{19} \mathrm{~m}^{-3}\right.$ and higher $)$. 

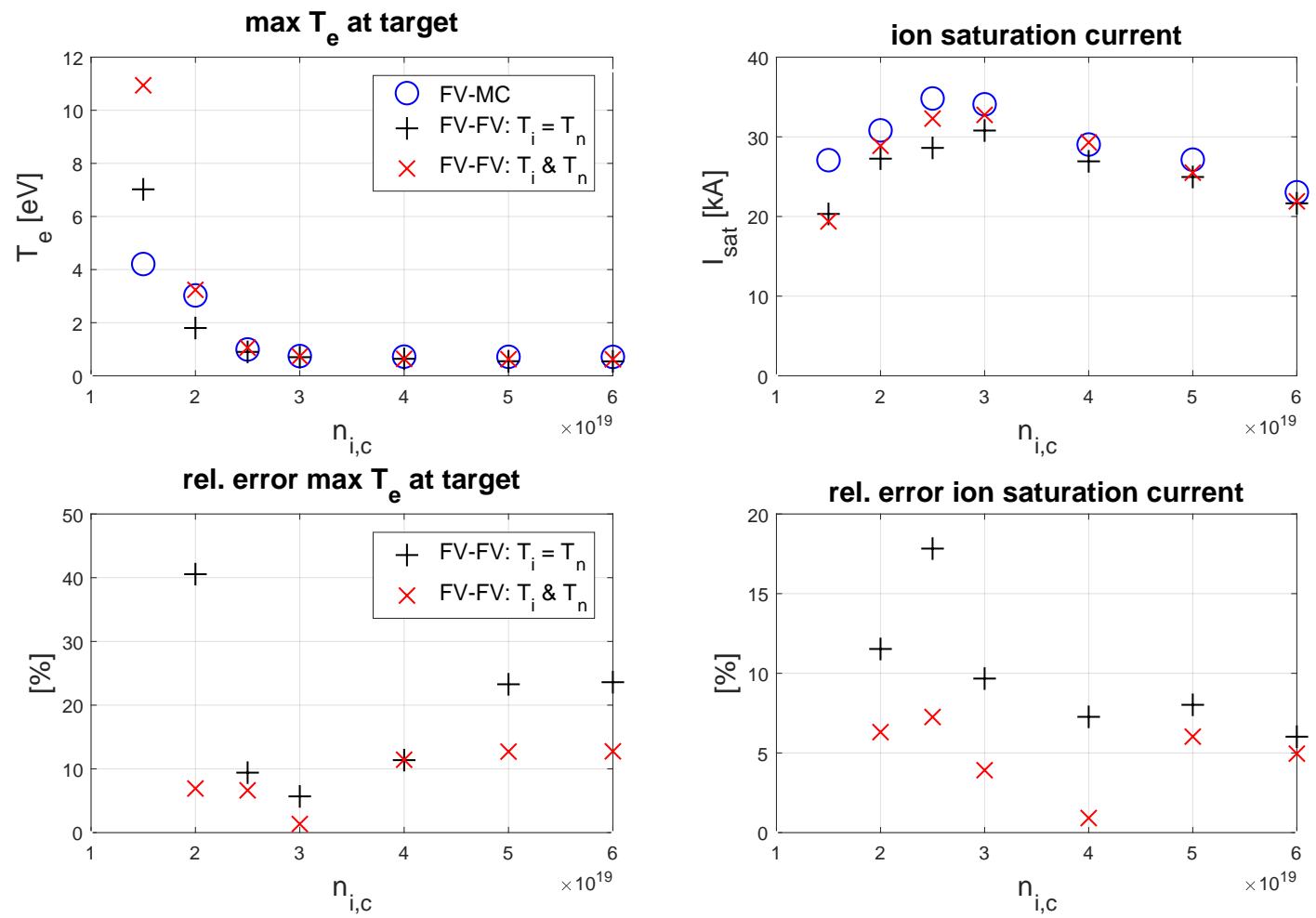

Figure 3: Maximum target electron temperature (top left) and total ion saturation current (top right) for a range of core ion densities, obtained with the Braginskii plasma model coupled to three different neutral models: the EIRENE MC code (FV-MC), the fluid neutral model with combined ion-neutral energy equation $\left(F V-F V: T_{i}=T_{n}\right)$ and the fluid neutral model with a separate ion and neutral temperature $\left(F V-F V: T_{i} \&\right.$ $\mathrm{T}_{\mathrm{n}}$ ). The bottom figures display the relative error compared to the FV-MC solution. In the bottom figures we omit the case with lowest core density for visual clarity.

\section{Conclusions and future work}

In this contribution, we have implemented a separate neutral energy equation in the SOLPS-ITER code, thereby completing the implementation of the advanced fluid neutral models developed by N. Horsten et al. into the code. When solving the coupled plasma-neutral system, the fluid neutral models presented in this paper offer an accurate approximation to the kinetic description for high-recycling and detached regimes, both with and without separate neutral energy equation. The separate neutral energy equation improves results for detached cases and slightly extends the validity range towards cases with lower core ion density. In subsequent work, we will integrate this advanced fluid neutral model with the 9-point stencil developed in Ref. 5 to correctly deal with the anisotropic transport in non-orthogonal geometries, to enable the use of the advanced fluid neutral models in realistic tokamak configurations. Future work will focus on the generalization of the fluid neutral models to include the effects of plasma drifts and molecular processes.

\section{Acknowledgements}

Parts of the work are supported by the Research Foundation Flanders (FWO) under project grant G078316N. Part of this work was carried out within the framework of the EUROfusion Consortium and has received 
funding from the Euratom research and training programme under grant agreement No. 633053. The views and opinions expressed herein do not necessarily reflect those of the European Commission. The computational resources and services used in this work were provided by the VSC (Flemish Supercomputer Center), funded by the Research Foundation Flanders (FWO) and the Flemish Government - department EWI. M. Blommaert is a postdoctoral research fellow of the Research Foundation - Flanders (FWO) and the Flemish Institute for Technological Research (VITO). Niels Horsten is a PhD fellow of the Research Foundation - Flanders (FWO).

\section{References}

[1] M Blommaert et al. "A spatially hybrid fluid-kinetic neutral model for SOLPS-ITER plasma edge simulations". In: Nuclear Materials and Energy 19 (2019), pp. 28-33.

[2] M Blommaert et al. "Implementation of a consistent fluid-neutral model in SOLPS-ITER and benchmark with EIRENE". In: Contributions to Plasma Physics 58.6-8 (2018), pp. 718-724.

[3] SI Braginskii. "Transport processes in a plasma". In: Reviews of plasma physics 1 (1965), pp. 205-311.

[4] W Dekeyser et al. B2-B2. 5 code benchmarking. Tech. rep. Forschungszentrum Juelich (Germany). Inst. fuer Energie-und Klimaforschung (IEK), 2011.

[5] W Dekeyser et al. "Implementation of a 9-point stencil in SOLPS-ITER and implications for Alcator C-Mod divertor plasma simulations". In: Nuclear Materials and Energy 18 (2019), pp. 125-130.

[6] GH Dunn. "Franck - Condon Factors for the Ionization of H2 and D2." In: The Journal of Chemical Physics 44(7) (1966), pp. 2592-2594.

[7] K Ghoos et al. "Accuracy and convergence of coupled finite-volume/Monte Carlo codes for plasma edge simulations of nuclear fusion reactors". In: Journal of Computational Physics 322 (2016), pp. 162-182.

[8] N Horsten, G Samaey, and M Baelmans. "Development and assessment of 2D fluid neutral models that include atomic databases and a microscopic reflection model". In: Nuclear Fusion 57.11 (2017), p. 116043.

[9] N Horsten et al. "Assessment of fluid neutral models for a detached ITER case". In: Nuclear Materials and Energy 12 (2017), pp. 869-875.

[10] RA Pitts et al. "Status and physics basis of the ITER divertor". In: Physica Scripta 2009.T138 (2009), p. 014001.

[11] D Reiter, M Baelmans, and P Boerner. "The EIRENE and B2-EIRENE codes". In: Fusion science and technology 47.2 (2005), pp. 172-186.

[12] ME Rensink et al. "Comparison of neutral gas models for divertor plasmas". In: Contributions to Plasma Physics 38 (1998), pp. 325-330.

[13] S Wiesen et al. "The new SOLPS-ITER code package". In: Journal of nuclear materials 463 (2015), pp. $480-484$.

[14] F Wising et al. "Simulation of Detachment in ITER-Geometry Using the UEDGE Code and a Fluid Neutral Model". In: Contributions to Plasma Physics 36.2-3 (1996), pp. 309-313. 